\title{
'Pugnatron'-like reaction in a patient with familial dystonia: torque induced motion analysis
}

\author{
E. GEOFFREY WALSH
}

\author{
From the Department of Physiology, University of Edinburgh, Edinburgh
}

SYNOPSIS A patient is described who suffered from an unusual extrapyramidal syndrome involving both legs and one arm. In the affected parts there was an extreme degree of rigidity. Measurements have been made of the motion that occurred as a result of applying force to the left foot. The torque was provided by a printed motor. The effect of a maintained plantar flexing force was anomalous for the foot moved to a dorsiflexed position. A model illustrating possible principles of feedback control - the Pugnatron-is described.

When force is applied to a limb it normally moves in the direction in which the force is applied. The extent to which it moves varies with the amount of the force and its duration. The degree of motion attained in a spastic limb is in general limited by the operation of viscous and elastic forces in the active muscles and often also by enhancement of muscular activity by reflex mechanisms. Most of these features are almost self-evident during a clinical examination and can be verified by concomitant electromyography.

For the investigation of muscle tone it has been customary to displace the limb by some mechanical contrivance and measure the resulting force (Walsh, 1971a). The development in recent years of a new electrical instrument has enabled the problems of muscle tone to be viewed from a different angle. The device is the 'printed motor' (Evans, 1972) and for the first time provides a convenient arrangement for applying force to a limb in a versatile manner for the force, or 'torque' since rotation is being considered, varies according to the current supplied. Torque generators of this type have been used to study the servo-control of posture at the wrist (Walsh, $1970 \mathrm{a} ; 1973$ ) and the control of the jaw (Walsh, 1970c). They have also been used to study tremor (Walsh, 1969), the cogwheel effect (Walsh, 1970b), clonus (Walsh, 1971b; Harris and Walsh, 1972a), reflexes in paraplegia (Harris and Walsh, 1972b), and the activation of rigidity when a skilled movement is attempted
(Gillingham et al., 1972; Dinakar and Walsh, 1973). One set of measurements has been obtained during cramp of the calf muscles resulting from strenuous cycling (Muir et al., 1970).

By varying the force and measuring the resultant position changes, data are obtained which are quite different from those using the traditional method in which the position is varied and the force measured. Both systems can give useful information according to the nature of the problem under investigation. The importance of choosing force rather than position as the independent variable for certain observations was first seen by Roberts (1963). The data of the two systems are not interconvertible and, by varying the torque applied to a limb while recording the mechanical and electromyographic events that follow, a great deal of information can be directly obtained about the control systems that are operative. The method may be called that of a 'torque induced motion analysis'. The technique has now enabled a form of hypertonicity to be defined that does not seem to have been recognized hitherto ${ }^{1}$; it is with this question that the present report is concerned.

Some years ago electrical engineers found that by the application of certain types of feedback to servo controlled motors interesting new properties were obtained and the system became load seeking. A system of this type has been called a

1 See, however, an account of the phenomenon described as 'gegenhalten' by Kleist (1927). 
Pugnatron, a name apparently coined by Professor F. C. Williams, FRS.

A motor controlled appropriately by a combination of negative and positive feedback loops will increase rather than decrease its speed when loaded (Williams and Uttley, 1946). With the speed set to zero with no load, the motor starts to turn when a load is applied in such a direction as to do work. In one observation a piece of string was wrapped round the pulley on the motor shaft and fitted with a bucket at each end. When a weight was placed in one of the buckets it was hauled up. Alternatively with the motor fitted to a small truck placed on a slope, the vehicle invariably climbed to the top irrespective of which way it was facing. Electric motors are sources of mechanical energy as are muscles and the question arises as to whether any neural pathways behave in a manner similar to the servo systems described above. Some consideration of the possibilities in this direction were mentioned at a demonstration of a different type of Pugnatron to the Physiological Society
(Walsh, 1968). This was a theoretical study involving a model in which possible biological implications were briefly considered. This Pugnatron is considered in further detail in the present paper. A patient has now been studied who has been found to have reflex responses of the Pugnatron type.

\section{METHOD}

The reflexes at the foot were investigated by 'torque induced motion analysis' (Fig. 1). The patient lay on her left side with her left foot attached to the spindle of a large printed motor (type G $16 \mathrm{M} 4$ ). The motion was of dorsiflexion and plantar flexion, the axis of motion being vertical. It was necessary to splint the foot firmly within a U-shaped piece of metal to ensure that motion was not lost, for the inverted position of the foot made a simple pedal type arrangement unsatisfactory. The spindle of the motor is double ended and, while the upper end drove the foot, the lower end was coupled to an 'infinite resolution conductive plastic potentiometer'. Byo ' varying the current through the transistor amplifie controlling the motor, corresponding forces of of

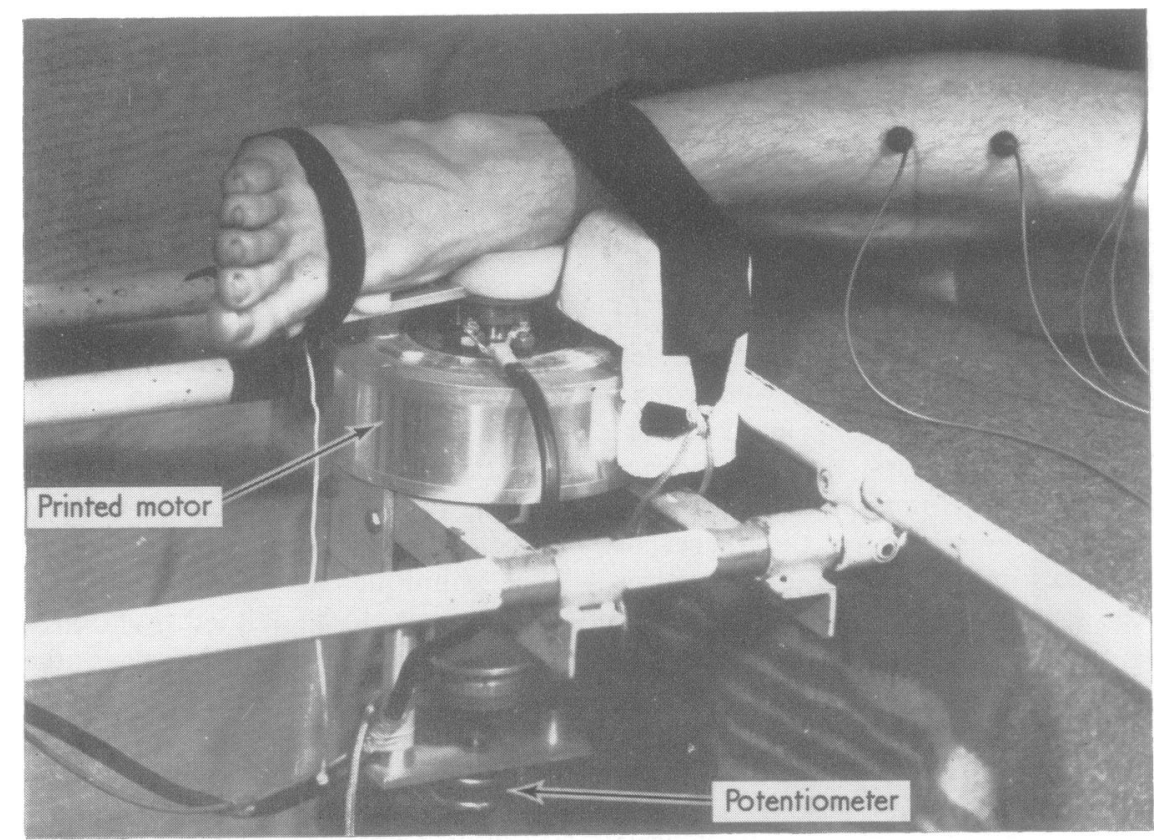

FIG. 1. Normal right foot attached to the apparatus. Patient lies on couch, foot over end. Large tubular metal frame is for stability. Ankle bandaged to shaped block of expanded polystyrene by Velcro. EMG electrodes seen over tibialis anterior. 
plantar- or dorsiflexion were produced. A Mingograph ink jet recorder was used to monitor the responses. The current through the motor corresponded to the torque applied. The plastic potentiometer gave a record of the alterations in the position of the foot. This signal was differentiated by an operational amplifier to give a velocity trace. Surface electromyography was performed using suction cup rubber electrodes filled with jelly and attached over the gastrocnemius and tibialis anterior muscles. Acceleration was recorded by strapping to the foot an (Electro Mechanisms) piezo-electric angular accelerometer (type QZR). This fed a FET amplifier (Ancom 15A-7) with a $500 \mathrm{M} \Omega$ input resistor.

CLINICAL HISTORY Mrs. C.C., 31 year old housewife, had developed tonic inversion of the right ankle at the age of 20 years during her first pregnancy. This progressed and interfered with walking, spread first to the right upper limb and then to the left leg. For the last two years she has been confined to a wheelchair. Apart from inability to walk, the dystonia of the three limbs had caused relatively little impairment of other activities and she continued to run her house. She had some spinothalamic sensory impairment and imperfect bladder control. For the last six years, she was liable to sudden powerful opisthotonic spasms which occurred during sleep and wakefulness and were liable to throw her from her wheelchair. This had led to three admissions to the local Head Injuries Unit.

In the course of repeated hospital admissions it had been shown that the cerebrospinal fluid was normal, and myelography, pneumoencephalography, and electroencephalograms had revealed nothing amiss.

Two other members of the family suffered from an unusual neurological condition: her mother's first cousin Mr. J.L. aged 57 years, and his sister Mrs. A.McL., aged 65 , have been afflicted by a slowly progressive paraplegia for eight years and 23 years, respectively.

On examination, intellect and cranial nerves were normal, as was the left upper limb. The other three limbs were held in tonic adduction and inversion, more so at the periphery of the limbs. This reduced facility of voluntary movements to a variable degree. She had normal position and vibration sense, but pain and temperature were impaired below the fourth cervical dermatome on the right side and the fourth dorsal on the left.

Tendon jerks were brisk and symmetrical, Hoffman's sign was positive and the plantar reflexes extensor, but both these abnormal reactions exhibited a curious delay.

The disorder of tone disappeared during sleep and general anaesthesia.

A stereotaxic operation on the left side reduced the rigidity of the limbs on the right side of the body. Three months later (February 1973) the observations recorded in this paper were undertaken and afterwards a stereotaxic operation was performed on the right side. The rigidity of the left foot was then relieved and the Pugnatron-like activity abolished temporarily. When tested in October 1973 both phenomena were again present.

Taken as a whole, the observations are consonant with an organic disorder of the brain but the nature, extent, and position of the lesion or lesions is not known.

\section{RESULTS}

APPLICATION OF A STEADY FORCE When the foot was pushed down by hand the impression was

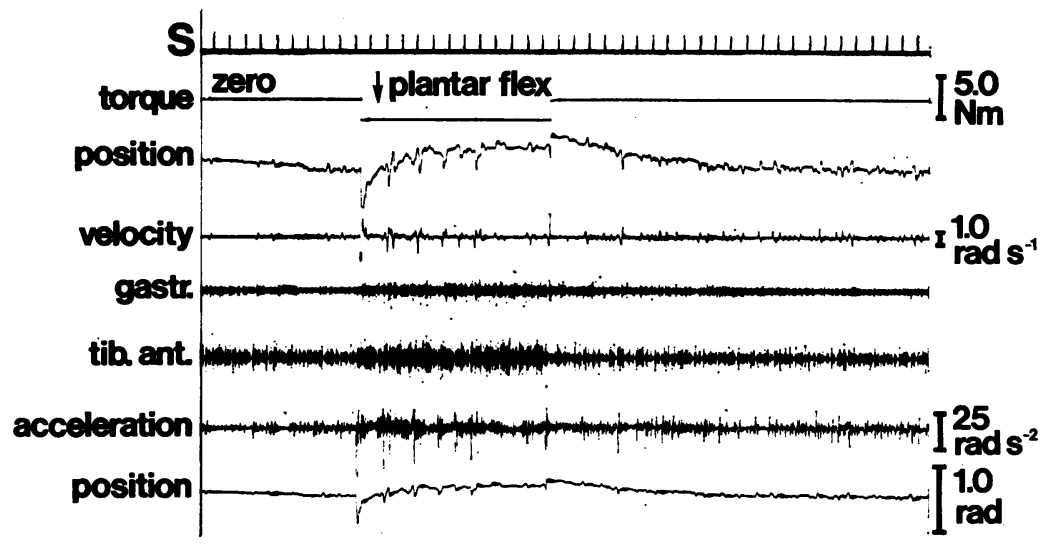

FIG. 2. Motion in response to steady plantar flexing force. Final position is one of dorsi-flexion. Position changes recorded in two traces at different sensitivities. 

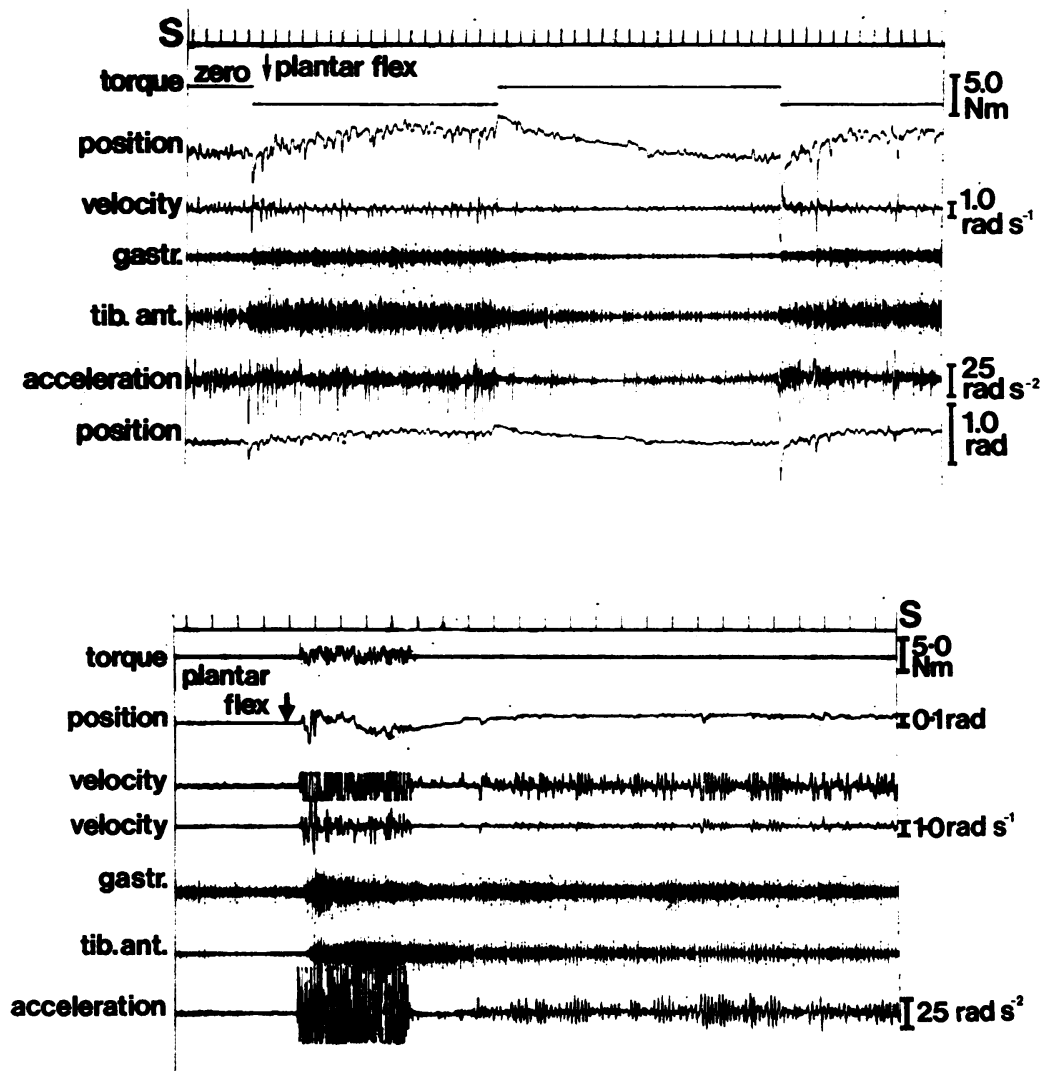

FIG. 3. Tremulous motion to a dorsiflexed position on applying a force tending to plantar flex foot. Two examples.
FIG. 4. Changes provoked by applying random forces (top line). EMG changes are prolonged, slowly subside. Tremor appears after a short latency, a phenomenon seen in other records with the random force stimulus. Velocity recorded in two traces at different sensitivities Tibialis anterior activity is grouped at frequency of tremor. gained that the displacement was being actively resisted in that extra force was being brought in by the dorsiflexors. This conclusion is made clear by the type of record that was obtained when a steady torque was applied in a plantar flexing direction (Fig. 2). At the moment that the force was switched on, the foot, hitherto in equilibrium, naturally started to move in the direction of the applied force. This movement was, however, rapidly checked and within a second or so the foot had reestablished its old position; with the further passage of time dorsiflexion continued, a plateau being reached after several seconds. It is clear then that the applied torque had been more than matched by a force produced within the muscles of the leg. The effect may be described not as action and reaction but perhaps 'action and super-reaction'. Throughout the procedure, the electromyographs of both channels, but especially that from tibialis anterior, showed increased activity. When the force was withdrawn, the foot sprang further into dorsiflexion and then gradually returned towards a less abnormal position. Two additional sets of observations are shown in Fig. 3. Altogether, the main features of this effect were seen in 11 trials. The tendency of the foot to plantarflex was repelled and as the patient had no useful power in that leg, the response was presumably a reflex.

EFFECT OF RANDOM, OF BRIEF, AND OF RHYTHMIC FORCES The limb was liable to abrupt increases of muscle action after a brief period in which random forces were applied through the printed motor (Fig. 4). This was associated with the development of a tremor at about $7 \mathrm{~Hz}$. The increased muscle action was clearly seen in both electromyographic traces and long outlasted the precipitating stimulus. The potentials in the trace from tibialis anterior seemed to be grouped 
at the frequency of the tremor, while those from gastrocnemius were steady. This type of response was seen in eight trials. The reaction was provoked by forces of quite mild intensity. It was evidently easy to provoke an exacerbation of the dystonia which was only slowly and with difficulty checked.

Tremor of the foot was also provoked by brief pressure through a finger applied to the gastrocnemius muscle. The tremor was of long duration and the effect was seen in five trials.

The resonant frequency of the foot was determined by the use of rhythmic sinusoidal torques. The frequency of the forces was increased exponentially. The value obtained $-10 \mathrm{~Hz}$-is substantially higher than that of a normal foot and is an expression of the rigidity (Fig. 5).
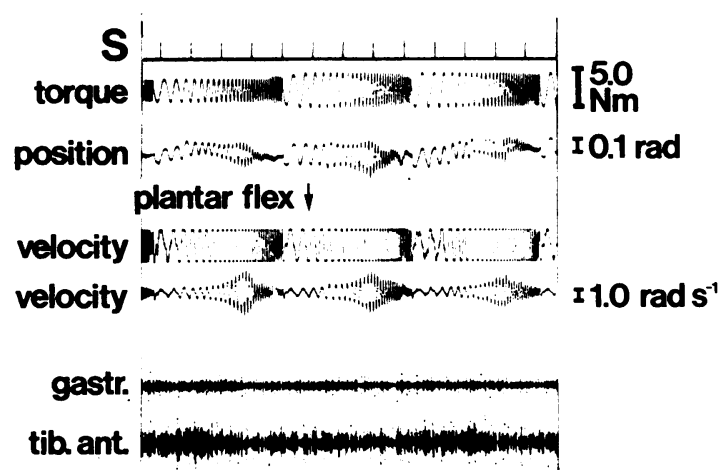

FIG. 5. Force varied in frequency, motion recorded. Upper velocity trace: too sensitive and irrelevant with waves clipped; lower velocity trace: maximum response at the 'resonant frequency'. Both gastrocnemius and tibialis anterior show activity. Resonant frequency, pathologically high due to co-contraction of muscles. Little modulation of electrical activity by the applied force.

Thirty-four frequency sweeps were recorded using the automatic programming system with which the apparatus has been equipped.

THE PUGNATRON-A MODEL SHOWING A SIMILAR RESPONSE (Fig. 6) The model derives its power from a steel drum which is rotated at low speed by a geared electric motor. Around the drum is wrapped two-and-a-half turns of a piece of

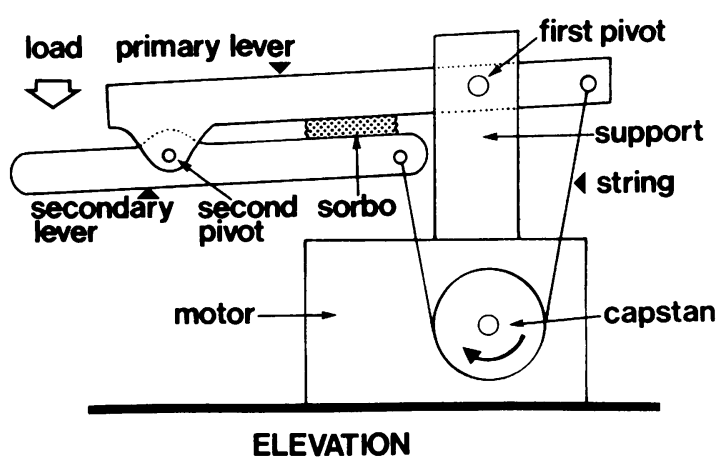

FIG. 6. Pugnatron. Equilibrium is reached when the force exerted by the capstan on the string attached to the primary lever is adequate to bear the weight of the system. This is attained when the string from the secondary lever is relatively slack.

nylon cord. The drum accordingly acts as a capstan and when the 'trailing' end of the string is pulled on the effect is magnified by the mechanical amplifier properties of this type of system and the other end of the string exerts a pull many times that of the original force. The cord runs from one end of the 'primary lever' round the capstan to the 'secondary lever'. The primary lever carries the secondary lever, the two being connected by a pivot. A spring is connected between the two levers or a piece of resilient plastic foam or sorbo rubber may be used. When a load is applied to the system, the string attached to the secondary lever is tensed and there follows accordingly an intensified pull on the primary lever. The apparatus thus pushes against the applied load. For any given weight, within the limits of the apparatus, there is an equilibrium point; for, as the capstan operates, the string tends to become slacker, as its attachment to the primary lever is further from the first pivot than is its attachment to the secondary lever. To a brief tap there is a jerk-like response reminiscent of a tendon reflex. If instead of being pressed down the system is supported the capstan goes slack and ceases to act until the surface is withdrawn. (The postural apparatus of a human being normally relaxes when a support is available, it comes immediately into action when this is removed.) If the end of the instrument is fitted with a brush which is brought into contact with a piece of cardboard, rhythmic up and 
down 'painting' movements are made. If the end of the instrument touches flexible material, such as polyurethane foam, it will make rubbing movements. A double acting Pugnatron has been made which reacts not only to downward directed but also to upward forces. If it meets a substantial force it will accordingly struggle. Friction pads are desirable between the two levers, but for a simple model it is sufficient that there should be some friction in the second pivot. With no damping, the model oscillates. This is especially true when the secondary levertaking account of any weight that may be fixed to it-is poorly balanced about its pivot.

If the load is applied closer to the second pivot than indicated in Fig. 6 the responses are eventually lost. There is a point at which the application of a load makes no difference to the equilibrium position. If the system were regarded as a simple servo system it would be regarded as having infinite gain, but as stable. It is not, however, a simple servo, for there are two effects. First the load is sensed by compression of the sorbo between the two levers and this causes the capstan to work harder. Second, owing to the geometry of the string attachments (vide supra), there is a situation resembling negative position feedback. The action of the Pugnatron depends both on the tension feedback, on the position effect, and for stability on dynamic balancing assisted by some damping. In a biological correlate corresponding arrangements may be expected.

If the weight is placed even closer to the pivot than has been considered, the system is lowered rather than raised by its application. In these circumstances, the apparatus mimics a stretch reflex, the gain varying according to the exact position at which the load is applied. The stretch reflex may be considered a special case of the Pugnatron.

The device illustrates a principle, for positive feedback is involved when the secondary lever comes into contact with a stiff material. The harder the capstan works, the greater the effect, up to the limit of the instrument. It cannot, however, be said that positive feedback is exhibited to a steady force such as is presented by a weight. Evidently the type of servo system to be considered depends upon the external situation to a degree as important as the arrange- ment of the internal components. Finally, it may be noted that operational amplifiers are now frequently being used with multiple feedback pathways and some of these systems may be worthy of study by physiologists in so far as the arrangements mimic biological systems.

\section{DISCUSSION}

It is not known where the receptors lie for the reflexes seen in the patient. It is clear that the applied force was being monitored and was giving rise to the muscle contraction. Golgi tendon organs monitor tension but traditional neurophysiological concepts point to them as having an inhibitory rather than an excitatory effect on their muscles. The muscle spindles will act as tension receptors under certain circumstances. It would be necessary for the intrafusal fibres to contract to the same degree as the main muscle fibres with which they lie in parallel. Finally, it is possible that cutaneous or subcutaneous receptors might be responsible. There is, of course, a positive supporting reaction in normal infants and this may have some features in common with the reaction under discussion but the muscles involved are wholly dissimilar.

Pugnatron-like responses do not seem to have been described in neurophysiological experiments on animals. If they were present, the animal might be struggling and anaesthetics and relaxants might be administered until it was in a passive condition. By the nature of the usual arrangements for neurophysiological observations, the likelihood of observing the responses is not high. Furthermore, the method of torque induced motion analysis used in the present investigation has not been used for animal investigations.

Pugnatron-like reactions may possibly be important in some common actions where the neuromuscular system interacts with the environment. Meeting resistance the Pugnatron heaves against it. Only further study will show whether this type of reaction has relevance to standing, pushing, and rhythmic movements such as scratching, kicking, rowing, sucking, and walking. Traditional neurological thought would speak of the release of a reflex that is normally kept carefully in check by inhibition. Powerful muscular reactions are liable to be brought into 
play by Pugnatron-like pathways. Continuous inhibitory processes would normally be operative to be released only temporarily under rather special conditions. How far the responses are the basis of other states of disturbed muscle tone remains to be seen.

The work is being supported by a Royal Society (Government) grant, and by Roche Products Ltd. The apparatus, including the Pugnatron, was constructed by Mr. G. Wright, who also provided skilled assistance during the recordings. The patient was under the care of Dr. E. Jellinek, to whom the author is indebted for clinical details, and of Professor F. J. Gillingham.

\section{REFERENCES}

Dinakar, I., and Walsh, E. G. (1973). Parkinsonism-two types of activation. Journal of Physiology, 231, 31-32P.

Evans, R. M. (1972). The printed circuit motor. Electronics Today International, 1, no. 2, May, 14-18.

Gillingham, F. J., Tsukamoto, Y., and Walsh, E. G. (1972). Treatment of rigidity. In Parkinson's Disease, vol. I, pp.93114. Edited by J. Siegfried. Huber: Bern.

Harris, P., and Walsh, E. G. (1972a). The use of torque generators to study clonus and disturbances of muscle tone. Paraplegia, 9, 228.

Harris, P., and Walsh, E. G. (1972b). Simultaneous electrical and mechanical recording from postural muscles in a paraplegic patient. Paraplegia, 9, 229-230.
Kleist, K. (1972). Gegenhalten (motorischer Negativismus), Zwangsgreifen [und Thalamus:Opticus. Monatsschrift für Psychiatrie und Neurologie (Berlin), 65, 317-396.

Muir, A. L., Percy-Robb, I. W., Davidson, I. A., Walsh, E. G., and Passmore, R. (1970). Physiological aspects of the Edinburgh Commonwealth Games. Lancet, 2, 11251128.

Roberts, T. D. M. (1963). Rhythmic excitation of a stretch reflex, revealing (a) hysteresis and (b) a difference between the responses to pulling and to stretching. Quarterly Journal of Experimental Physiology, 48, 328-345.

Walsh, E. G. (1968). Model of a load-seeking reflex-a 'Pugnatron'. Journal of Physiology, 197, 57-59P.

Walsh, E. G. (1969). Interference with the tremor of Parkinsonism by the application of a rhythmic force. Journal of Physiology, 202, 109-110P.

Walsh, E. G. (1970a). Tremor of the wrist induced by positive velocity feed-back. Journal of Physiology, 207, 16$17 \mathrm{P}$.

Walsh, E. G. (1970b). Oscillatory transients in the response to an abrupt change of force at the wrist in Parkinsonism. Journal of Physiology, 209, 33-34P.

Walsh, E. G. (1970c). Movements of the jaw resulting from the application of external forces. Journal of Physiology, 210, 179-180P.

Walsh, E. G. (1971a). Studying muscle tone. Developmental Medicine and Child Neurology, 13, 113-114.

Walsh, E. G (1971b). Ankle clonus-an autonomous central pace-maker? Journal of Physiology, 212, 38-39P.

Walsh, E. G. (1973). Motion at the wrist induced by rhythmic forces. Journal of Physiology, 230, 44-45P.

Williams, F. C., and Uttley, A. M. (1946). The velodyne. Journal of the Institution of Electrical Engineers, 93, 12561274. 